\title{
Tensor Effects in Dipole Excitations at Finite Temperature
}

\author{
E. YÜKSEL AND K. BOZKURT \\ Physics Department, Yildiz Technical University, 34220 Esenler, Istanbul, Turkey
}

\begin{abstract}
Tensor effects in the dipole excitation of neutron-rich ${ }^{68} \mathrm{Ni}$ nucleus are investigated in the framework of SkyrmeHartree-Fock plus random phase approximation at finite temperature. We calculate isovector giant and pygmy dipole strengths with finite temperature random phase approximation by using different tensor correlations. The effect of both tensor and finite temperature on the giant dipole resonance-pygmy dipole resonance energy region is analysed. Pygmy dipole resonance calculations with different proton-neutron tensor coupling constants are also compared with the experimental results.
\end{abstract}

DOI: 10.12693/APhysPolA.123.320

PACS: 21.60.-n, 21.60.Jz., 24.30.Cz

\section{Introduction}

As one of the most known collective motion of nuclei, giant dipole resonance (GDR) is defined as $1 \mathrm{p}-1 \mathrm{~h}$ excitation of nuclei and the resonance energy is found typically between $12-25 \mathrm{MeV}$ [1]. Formation of low-energy dipole excitations are also studied by scientists in neutron rich nuclei. This low-energy mode is forming below GDR region (between 4 and $12 \mathrm{MeV}$ ) because of the neutron excess and known as pygmy dipole resonances (PDR).

The changes in the width and energy of giant resonances are an important subject. One of them is the finite temperature effect which changes the shape of nuclei with the thermal fluctuations. Both theoretical and experimental works based on finite temperature effect have been performed so far [2-9]. The main outcome is that nucleus becomes deformed, the GDR width increases and position changes slightly especially between $T=0-3 \mathrm{MeV}[2-9]$.

In recent years, another important topic on the nuclear structure and collective response of nuclei is the tensor effect. Addition of tensor parameters to the Skyrme parameters gives rather good results to explain nuclear structure and collective response of nuclei [10-13]. Both finite-temperature and tensor effect has some consequences on the shell evaluation and collective motion of nuclei. In this framework, the development of calculations with both finite-temperature and tensor effect is necessary to explain the collective response of nuclei from different perspectives.

In this paper, we investigate pygmy and giant dipole excitations in neutron-rich ${ }^{68} \mathrm{Ni}$ nucleus using the different Skyrme tensor parameters at finite temperature. We use the mean-field theory and employ random phase approximation (RPA) in coordinate space to calculate the isovector pygmy and giant dipole resonances at zero and finite temperature.

\section{Model}

Finite-temperature (FT) Green's function $G_{0}$ is given by

$$
\begin{aligned}
& G_{0}\left(\boldsymbol{r}, \boldsymbol{r}^{\prime}, \omega\right)=\sum_{\mathrm{ph}} \phi_{\mathrm{p}}^{*}(\boldsymbol{r}) \phi_{\mathrm{p}}\left(\boldsymbol{r}^{\prime}\right) \\
& \quad \times\left[\frac{f_{\mathrm{p}}-f_{h}}{E_{\mathrm{p}}-E_{h}-\omega+\mathrm{i} \eta}+\omega \rightarrow-\omega\right] \phi_{h}^{*}(\boldsymbol{r}) \phi_{h}\left(\boldsymbol{r}^{\prime}\right),
\end{aligned}
$$

where $f_{\mathrm{p}, \mathrm{h}}=1 /\left(1+\mathrm{e}^{\beta E_{\mathrm{p}, \mathrm{h}}}\right)$ is the Fermi-Dirac occupation factor and $E$ is the single particle energy. Thus, FTRPA response function, which obeys a Bethe-Salpeter equation, is

$$
G_{\mathrm{FTRPA}}=G_{0}\left[1-G_{0} V_{\mathrm{ph}}\right]^{-1},
$$

where $V_{\mathrm{ph}}$ is the particle-hole Skyrme interaction and can be obtained as

$$
V_{\mathrm{ph}}(\boldsymbol{r})=V_{\mathrm{C}}(\boldsymbol{r})+V_{\mathrm{LS}}(\boldsymbol{r})+V_{\mathrm{T}}(\boldsymbol{r}),
$$

where $V_{\mathrm{C}}(\boldsymbol{r})$ is the density-dependent central interaction, $V_{\mathrm{LS}}(\boldsymbol{r})$ is the zero-range spin-orbit interaction and $V_{\mathrm{T}}(\boldsymbol{r})$ is the tensor interaction. Then using the Green function FTRPA the strength is obtained as [1]:

$$
\begin{aligned}
& S(E)=-\frac{1}{\pi} \operatorname{Im} \int \mathrm{d} \boldsymbol{r} \int \mathrm{d} \boldsymbol{r}^{\prime} F^{*}(r) \\
& \times G_{\mathrm{FTRPA}}\left(\boldsymbol{r}, \boldsymbol{r}^{\prime} ; \omega\right) F\left(\boldsymbol{r}^{\prime}\right)
\end{aligned}
$$

and $F$ is evaluated for a dipole excitation operator $F=$ $r Y_{10}$.

Finite temperature particle-hole and tensor Skyrme interaction can be obtained

$$
\begin{aligned}
& V_{\mathrm{ph}}=N_{0}^{-1}(T)\left(\tilde{F}_{0}+\tilde{F}_{0} \boldsymbol{\tau}_{1} \cdot \boldsymbol{\tau}_{2}+\tilde{G}_{0} \boldsymbol{\sigma}_{1} \cdot \boldsymbol{\sigma}_{2}\right. \\
& \left.\quad+\tilde{G}_{0}^{\prime} \boldsymbol{\tau}_{1} \cdot \boldsymbol{\tau} \boldsymbol{\sigma}_{1} \cdot \boldsymbol{\sigma}_{2}\right),
\end{aligned}
$$

where $\tilde{F}_{0}, \tilde{F}_{0}^{\prime}, \tilde{G}_{0}$ are the Landau parameters at the Fermi surface at finite temperature. This $\mathrm{p}-\mathrm{h}$ interaction also contains tensor components as [14]:

$$
\begin{aligned}
& H_{0}=N_{\mathrm{T}} \frac{\tilde{k}_{\mathrm{F}}^{2}}{5}\left(\alpha_{\mathrm{T}}+\beta_{\mathrm{T}}\right), \\
& H_{0}^{\prime}=N_{\mathrm{T}} \frac{\tilde{k}_{\mathrm{F}}^{2}}{5}\left(\alpha_{\mathrm{T}}-\beta_{\mathrm{T}}\right),
\end{aligned}
$$

where $N_{\mathrm{T}}=2 m \tilde{k}_{\mathrm{F}} / \pi^{2} \hbar^{2}$ is the density of states and $\tilde{k}_{\mathrm{F}}$ is the Fermi momentum at finite temperature. The tensor parts $\left(\alpha_{\mathrm{T}}, \beta_{\mathrm{T}}\right)$ are related to the zero-range exchange part of the central interaction and coefficients $(\alpha \beta)$. The details of the calculations can be found in Ref. [15]. 
For the tensor channel, Skyrme-SLy5-TIJ parameters are chosen. The tensor parameters are labeled as $T I J(I, J=1, \ldots, 6)$. To see the effect of tensor correlations in our finite temperature calculations, $T 44, T 43$, T64, T34, T46 parameters are chosen. The values of $\alpha_{\mathrm{T}}$ and $\beta_{\mathrm{T}}$ for T44, T43, T64, T34, T46, are $(40,170)$, $(-20,170),(40,290),(40,110),(160,170) \mathrm{MeV} \mathrm{fm}^{5}$.

\section{Results and discussion}

In this work, we present a mean-field description of isovector pygmy and giant dipole excitation in neutron-rich ${ }^{68} \mathrm{Ni}$ nucleus at zero and finite temperature. We use FTRPA to determine the nuclear response by using effective nucleon-nucleon Skyrme interaction SLy5 [16]. Our calculations are based on a Green function HF + RPA at zero and finite temperature. The interaction we use is based on FT+Landau-Migdal representation of the Skyrme interaction [17].

Results for the PDR region of ${ }^{68} \mathrm{Ni}$ nucleus within $\mathrm{HF}+\mathrm{RPA}$ calculations using the different kind of the Skyrme tensor parameters are compared in Fig. 1 with their experimental results at finite temperature. As we mentioned in the introduction, it has been known that GDR width increases and GDR components split with finite temperature effect. Recently, the low-energy strength of ${ }^{68} \mathrm{Ni}$ nucleus has been calculated with finite temperature relativistic RPA (FT + RRPA). It has been shown that GDR strength changes slightly and another low-energy strength is formed below $10.0 \mathrm{MeV}$ at $T=2 \mathrm{MeV}[6]$.

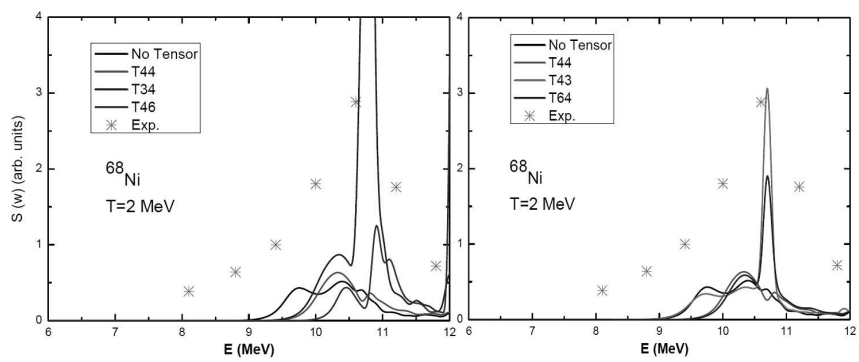

Fig. 1. Left part: the PDR strength function of ${ }^{68} \mathrm{Ni}$ with HF+RPA using T44, T34, T46 tensor parameters at $T=2 \mathrm{MeV}$. The normalized data are taken from [18]. Right part: the same calculations but for $T 43, T 64$.

In our calculations, with the inclusion of tensor interaction, low-energy strength increases for $T 43, T 64$ tensor parameters and gives much more narrower peaks at about $10.5 \mathrm{MeV}$ with respect to finite temperature calculations without tensor correlations. We find that the low-energy PDR results with and without tensor at finite temperature is closer to the experimental value of energy which is found nearly $11 \mathrm{MeV}[18]$. However for the PDR strength result with tensor interaction, like T34, we observe that the strength peak value is much larger than the corresponding experimental value. We have also analyzed PDR-GDR collectivity and energy using the $T 46$ and T64 tensor parameters at finite temperature as shown in Fig. 2. The effect of tensor parameters can be seen both in strength function and resonance energy in ${ }^{68} \mathrm{Ni}$ nuclei at zero and finite temperature in Fig. 2.

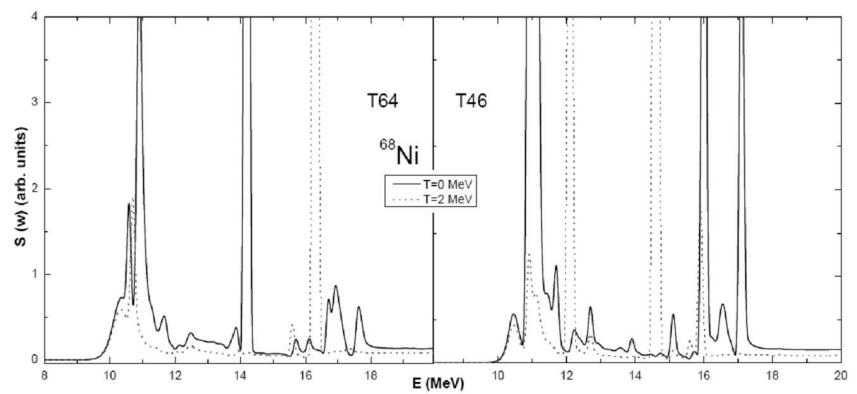

Fig. 2. The PDR-GDR strength functions of ${ }^{68} \mathrm{Ni}$ with $\mathrm{HF}+\mathrm{RPA}$ using the T46, T64 tensor parameters at zero and finite temperature.

We observe that the tensor contribution is more active in GDR for the different contributions of protons and neutrons to the tensor force. Tensor parameters with different proton-neutron contributions are also changing the PDR strength function results. Moreover, strong proton-neutron coupling (T64) gives rather good results at finite temperature in Fig. 2.

Giant and pygmy strength in the PDR-GDR energy region for finite and zero temperature results for the case of nuclei ${ }^{68} \mathrm{Ni}$ are also compared in Fig. 3. The PDRGDR strength function calculation results for the case of ${ }^{68} \mathrm{Ni}$ nucleus at zero and finite temperature are compared in Fig. 3 without tensor correlations.

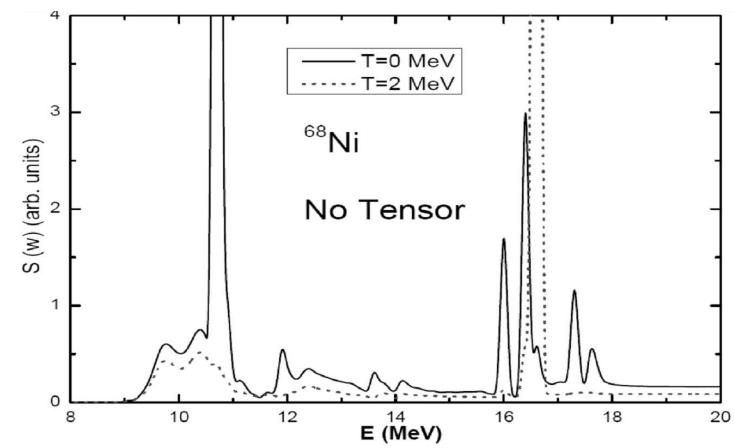

Fig. 3. The PDR-GDR strength functions of ${ }^{68} \mathrm{Ni}$ using $\mathrm{HF}+\mathrm{RPA}$ without tensor interactions at zero and finite temperature.

In Fig. 3 while the strength function decreases considerably, resonance energy remains the same in the PDR region. However, the inclusion of finite temperature increases strength function in GDR region and gives a clear peak. 


\section{Conclusion}

In summary, we calculated and compared the isovector giant and pygmy dipole strength in the PDR-GDR energy region in neutron-rich ${ }^{68} \mathrm{Ni}$ nucleus with different tensor parameters at finite temperature. We find that the PDR-GDR collectivity of this neutron-rich nuclei depends on the chosen proton-neutron or particle-like tensor terms at finite temperature. We show that both finite temperature and tensor interaction changes the collective response of ${ }^{68} \mathrm{Ni}$ nucleus. In particular, inclusion of tensor interaction to the finite temperature effect changes the response function.

\section{References}

[1] P. Ring, P. Schuck, The Nuclear Many Body Problem, Springer, New York 1980.

[2] E. Khan, N. Van Giai, M. Grasso, Nucl. Phys. A 731, 311 (2004).

[3] D. Vautherin, N. Vinh Mau, Nucl. Phys. A 422, 140 (1984)

[4] H. Sagawa, G.F. Bertsch, Phys. Lett. B 146, 138 (1984)

[5] E. Sahin, K. Bozkurt, M. Sirin, AIP. Conf. Proc. 1203, 11 (2010)

[6] Y.F. Niu, N. Paar, D. Vretenar, J. Meng, Phys. Lett. B 681, 315 (2009).

[7] A. Bracco, J.J. Gaardhoje, A.M. Bruce, J.D. Garrett, B. Herskind, M. Pignanelli, D. Barneoud, H. Nifenecker, J.A. Pinston, C. Ristori, F. Schussler, J. Bacelar, H. Hofmann, Phys. Rev. Lett. 62, 2080 (1989)
[8] E. Ramakrishnan, T. Baumann, A. Azhari, R.A. Kryger, R. Pfaff, M. Thoennessen, S. Yokoyama, Phys. Rev. Lett. 76, 2025 (1996)

[9] T. Baumann, E. Ramakrishnan, A. Azhari, J.R. Beene, R.J. Charity, J. E Dempsey, M.L. Halbert, P.-E. Hua, R.A. Kryger, P.E. Mueller, R. Pfaff, D.G. Sarantites, L.G. Sobotka, D.W. Stracener, M. Thoennessen, G. Van Buren, R.L. Varner, S. Yokoyama, Nucl. Phys. A 635, 428 (1998)

[10] G. Coló, H. Sagawa, S. Fracasso, P.F. Bortignon, Phys. Lett. B 646, 227 (2007)

[11] H. Sagawa, "Effect of Tensor Correlations on Single-particle and Collective States", arxiv/0910.5277v1

[12] Li-Gang Cao, G. Col, H. Sagawa, P.F. Bortignon, L. Sciacchitano, Phys. Rev. C 80, 064304 (2009)

[13] B.A. Brown, T. Duguet, T. Otsuka, D. Abe, T. Suzuki, Phys. Rev. C 74, 061303(R) (2006)

[14] L.G. Cao, G. Col, H. Sagawa, Phys. Rev. C 81, $044302(2010)$

[15] E. Yuksel, K. Bozkurt, Int. J. Mod. Phys. E 20, 2143 (2011)

[16] E. Chabanat, P. Bonche, P. Haensel, J. Meyer, R. Schaeffer, Nucl. Phys. A 635, 231 (1998).

[17] N. Van Giai, H. Sagawa, Phys. Lett. B 106, 379 (1981)

[18] O. Wieland, A. Bracco, Prog. Part. Nucl. Phys. 66, 374 (2011) 